\title{
LOCATION BASED INFORMATION SHARING SYSTEM FOR MOBILE DEVICES
}

\author{
Pallavi Gowdoor ${ }^{1}$, Satheesh Chandra Reddy $S^{2}$, Bharathi $\mathbf{M}^{3}$, Daina K K \\ ${ }^{1}$ M.Tech Student, Dept., of CS\&E, SJCIT, Chikkaballapura \\ ${ }^{2}$ Associate Professor and HOD, Dept., of IS\&E, SJCIT, Chikkaballapura \\ ${ }^{3}$ Associate Professor, Dept., of CS\&E, SJCIT, Chikkaballapura \\ ${ }^{4}$ Assistant Professor, Dept., of IS\&E, SKIT, Bangalore
}

\begin{abstract}
The mobile devices have become necessity of our lives. We use mobiles to share information to others. These mobile devices rely on the internet services provided by central ISP (internet service providers) to share information to other devices. But the services provided by internet service providers is not available in all the places and are expensive, have limited bandwidth. Information sharing between the mobile devices using the internet service requires the end device to have internet connection. To address these problems we provide a solution which enables information sharing between mobiles through inter device communication and makes use of information sharing technologies available on the mobile devices. To exhibit the advantages of our solution we are implementing interest based information sharing system in which the user interest will be expressed in small tags called strings. When two mobile devices come in contact they share the contents based on interest of each other.
\end{abstract}

Keywords: Content-based publish/subscribe, interest-driven information sharing, human network, bloom filter.

$* * *$

\section{INTRODUCTION}

MOBILE devices have become necessity of our lives. We need mobile to share information with others. These mobile devices rely on the internet services provided by central internet service providers to share information to other devices. The internet services providers is not available in all the places and are expensive, have limited bandwidth. Information sharing between the mobile devices using the internet service requires the end devices to have internet connection.

MOBILE devices need wireless technologies to communicate with one another via wireless infrastructures. Eg 3G/LTE GSM etc. But wireless infrastructure is not found everywhere. First due to unavailable network resources it fails in many circumstances.eg In a college campus, the Wifi and cellular connection can't be accessed properly because many user are accessing for the same channel at the same time. Second the inter-device communication os not supported in this wireless infrastructure.eg in a college campus, we can have excellent inter-device communication between nearby mobile devices.

Nowadays, most mobile applications are for information sharing; mobile devices are increasingly becoming the end points of information consuming. Evidence is that almost all existing smart phones and tablets are integrated with vendor-supplied music/video streaming services, and socialnetwork-based information sharing services are extremely popular on mobile devices. Given the existing architecture, however, they have to connect with central service providers, which would fail in many situations. Besides, this architecture can be inefficient in many scenarios. For instance, location-based chatting is more natural to implement in a peer-to-peer manner, so that nearby users can talk to each other directly.

In present scenario, mobile applications are used especially for information sharing; mobile devices are used as end points for information consuming. Mobile devices are integrated with different services provider for audio/video streaming services and social-network information sharing services. In existing architecture, in order to connect with each other mobile devices need central service providers, but it failed in many circumstances. So, this wireless architecture fails to use the available resources. In peer to peer manner, we can implement location based chatting, so we can communicate with nearby devices directly.

A "store-carry-forward" technique is used in the DTNs, which expands the capacity of mobile device. So in order to overcome with drawback of existing system, a new type of networking service called as human networks (HUNETS) is introduced. HUNETs, stands for human networks which is used for inter-device communication between the mobile devices. In existing system they use B-SUB, which stands for the bloom-filter-based publish/subscribe. B-SUB is planned in such a way it can be used for smaller to medium sized networks which consists of only few devices which can communicate where inter-device communications are restricted. B-SUB is applicable in different areas like college campus, in a small building, conference hall and so on.

The various features of B-SUB are listed below. First, BSUB uses the content based technique, in existing system. B-SUB selects the data based on their content instead of 
addresses. Second, B-SUB is more competent then publish/subscribe. Backup capacity and processors capacity is weak in mobile devices. These are the limitations of BSUB. The traditional technique consumes more bandwidth and memory capacity whereas HUNET overcome with these. High bandwidth and memory capacity is provided by HUNET.

B-SUB uses the technique of tag-based content description model. To compress content and user interests Bloom filters are used. An extensions of Bloom filter is Temporal Counting Bloom filter(TCBF) to encode tags, to get more efficient routing. Sometimes in TCBF the useless messages are forwarded to the nodes who are not interested in their content. TCBF suffers from false positives. Through the simulation studies the false positive in TCBF is analyzed. A HUNET is operated by human users. A HUNET consists of various wireless communication interfaces like Bluetooth and Wifi. In human network devices used only within short range communication. To enhance the communication for long range we can use the directional antenna. Human users in the HUNET are referred to as nodes. To improve the performance we can use the hot spots for non-humanoperated devices.

\section{RELATED WORK}

In [1] Delay Tolerant Networks Adopt a "store-carry-andforward" model in which message/information is stored in the source device till it finds a destination. They focus on delivering messages from a source to a destination. DTNs do not support interest-driven communication. DTN routing is based on the end-to-end model and has limited network resources. DTN's don't use interest driven communication. Memory usage will be more.

In [2], bloom filter is a data structure designed to tell rapidly whether an element is present in a set or not. Blooming filter publish-subscribe (B-Sub) system is used to check whether the message is in interest list or not. Publisher will broadcast all information whatever he is having to all. Subscriber will send only interested topic to others. B-Sub face problem of false positive.

In [3], User-Centric Data Dissemination in Disruption Tolerant Networks Distributed it satisfy user interests and forwards data only for nodes that are interested in data. Such nodes are "interesters". But drawback is interesters of data generally not known at priori at data source.

In [4],Epidemic routing is to distribute application messages to hosts called carries within connected portions of adhoc network. In this way messages are quickly distributed through connected portion of network. It relies upon carriers coming into contact with one another. Limitation difficult to design feedback mechanism. Wastage of network resources.

In [5], Cellular providers to offer quick and promising solutions. Straight forward solution is by increasing the cellular network capacity by adding more base station with smaller cell size such as piocells and femtocells. But limited to contact time.

\section{HUNETs}

Recently, inferring or sharing of mobile contexts has been actively investigated as cell phones have become more than a communication device. The main objective is to facilitate efficient information sharing between humans using mobile devices. A HUNET consists of portable devices that are equipped with wireless communication like $\mathrm{WiFi}$ or Bluetooth. These mobile devices are always carried and operated by human users, which gives name of human network.

In DTN's messages are exchanged based on store-and-carryforward technique where messages are stored and carried from source to destination, whereas in HUNET messages are exchanged based on interest of the users. In Bloom filter technique strings where used to check the whether messages to be forward or not. But bloom filters do not kept the counter. To over with this problem counting bloom filter where used to insert the tag along with counter and exchange messages. But existing strings couldn't be deleted so to overcome with this technique Temporal counting bloom filters(TCBF) where used. TCBF does not delete the string, but counter value will be updated by setting counter value to zero. In HUNET we are using the TCBF concept to exchange messages between the users.

A novel data structure, called Temporal Counting Bloom Filter (TCBF), is proposed to perform content based networking tasks. The TCBF's novelty is that it is able to handle temporal operations, which are not supported in the classic Bloom filter $(\mathrm{BF})$ and are crucial to the success of forwarding messages in HUNETs. TCBF is used to encode users' interests and embed routing information.TCBF are mainly used in order to do tag based Content description. If two interester $\mathrm{A}$ and $\mathrm{B}$ want to communicate the messages with each other at same time it cannot be done due to restriction of bandwidth, without the TCBF, only one of them get the chance to exchange messages. If we compress both can exchange messages easily. The TCBF servers this by matching the content instead of string matching.

\section{PROPOSED APPROACH}

B-SUB has two components: content representation and pub/ sub routing. B-SUB employs the tag-based content description model. The contents of messages and the interests of users are identified by tags, which are strings that summarize the topics of the message. They are stored in TCBFs, which are then used as probabilistic hints for forwarding messages. In this Paper we use TCBF in order to check the interested users and send messages to those who are connected based on interest. It's mainly based on interdevice communication.

\subsection{Tag Based}

Tag based content description is involved in B-Sub. In this paper we are using the tag based content description using TCBF. If we have any new messages to add first we need to add content and tag of messages. Then only we can 
communicate the messages with end devices. If any nearby devices are available we start exchanging the messages by exchange TCBF. Later messages are forward to other devices.

\subsection{Interest Propagation}

In B-SUB, TCBFs are used to compress users' interests. A user stores its own interests in a TCBF, which is called the genuine filter. A broker stores the interests collected from other users in another TCBF called the relay filter. TCBFs serve as a "compressed" matching hint for delivery. When two devices, say A and B meet, they first exchange the $\mathrm{TCBF}$ s that contain their genuine interests and relay interests. A then merges Bs genuine interests with its own relay interests using the A-merge; and merges Bs relay interests with its own relay interests using the M-merge. Note that all operations are performed on the TCBFs instead of the raw strings that are corresponding to the interests.

\subsection{Routing and Forwarding}

In routing and forwarding the preferential query is used by nodes to select forwarders for the buffer messages. The only data that is needed in the forwarding of messages is the TCBFs that encode the interests. The only operations performed are hashing and table lookup.

When two devices A and B meet, they exchange their relay interests and genuine interests encoded in TCBFs. At first devices exchange the genuine interested messages and forward to other devices. A then examines Bs relay filter to determine which other messages should be forwarded to B. A maintains a table of the preference values of all of the buffered messages, which is called a preference table. For each of the messages that have not been forwarded to B, A performs a preferential query of the message's tag to the relay filter of $\mathrm{B}$, and then compares the obtained preference value to the one associated with the message in the preference table. If $\mathrm{Bs}$ preference value is larger, the message is forwarded to $\mathrm{B}$, and the preference value of the message in the preference table is updated to Bs preference value. Otherwise, the message will not be forwarded. The same operations are also performed by $\mathrm{B}$.

The age of the messages in message buffer are based on when message was generated. The forwarding operation is carried based on newest to oldest messages. Once the buffer is overflowed all the older messages are deleted from the buffer.

\section{CONCLUSION}

In this paper, we presented B-SUB, an interest-driven information sharing system for mobile devices. B-SUB employs content-based networking to achieve infrastructureless communication between mobile devices. Specifically, BSUB employs a tag-based content description model. A novel data structure, the TCBF, is invented to compress user interests and guide content routing. The use of TCBF reduces the memory and bandwidth consumption of B-SUB.

\section{REFERENCES}

[1]. K. Fall, "A Delay-Tolerant Network Architecture for Challenged Internets," Proc. Conf.Applications, Technologies, architectures, and Protocols for Computer Comm.

[2]. Y. Zhao and J. Wu, "B-Sub: A Practical Bloom-FilterBasedPublish-Subscribe System for Human Networks," Proc. Int'l Conf. Distributed Computing (ICDCS '10), pp. 634-643, 2010.

[3]. "User-Centric Data Dissemination in Disruption Tolerant Networks Distributed Computing Systems" (ICDCS '10), pp. 634-643, 2011

[4]. C. Liu and J. Wu, "An Optimal Probabilistic Forwarding Protocol in Delay Tolerant Networks," Proc. MobiHoc '09, pp. 105-114, 2009.

[5]. Y. Li, G. Su, P. Hui, D. Jin, L. Su, and L. Zeng, "Multiple Mobile Data Offloading through Delay Tolerant Networks," Proc. Sixth ACM Workshop Challenged Networks, 2011.

[6]. Y. Li, Z. Wang, D. Jin, L. Zeng, and S. Chen, "Collaborative Vehicular Content Dissemination with Directional Antennas," vol. 11, no. 4, pp. 1301-1306, Apr. 2012.

[7]. R. Zhang, J. Shi, Y. Zhang, and J. Sun, "Secure Cooperative Data Storage and Query Processing in Unattended Tiered Sensor Networks," Selected Areas in Comm., IEEE J., vol. 30, no. 2, pp. 433-441, Feb. 2012.

[8]. R. Zhang, J. Shi, and Y. Zhang, "Secure Multidimensional Range Queries in Sensor Networks," Proc. ACM MobiHoc '09, pp. 197-206, 2009.

[9]. R. Zhang, J. Shi, Y. Zhang, and J. Sun, "Secure Cooperative Data Storage and Query Processing in Unattended Tiered Sensor Networks," Selected Areas in Comm., IEEE J., vol. 30, no. 2, pp. 433-441, Feb. 2012.

[10]. R. Zhang, J. Shi, and Y. Zhang, "Secure Multidimensional Range Queries in Sensor Networks," Proc. ACM MobiHoc ’09, pp. 197-206, 2009.

[11]. Y. Zhang and S. Zhong, "A Privacy-Preserving Algorithm for Distributed Training of Neural Network Ensembles," Neural Computing and Applications, pp. 1-14, DOI: $10.1007 / \mathrm{s} 00521-012-1000-8$.

[12]. W. Gao and G. Cao, "User-Centric Data Dissemination in Disruption Tolerant Networks," IEEE INFOCOM, pp. 3119-3127, Apr. 2011 